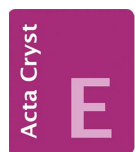

CRYSTALLOGRAPHIC COMMUNICATIONS

ISSN 2056-9890
Received 3 December 2018

Accepted 31 January 2019

Edited by $\mathrm{H}$. Stoeckli-Evans, University of Neuchâtel, Switzerland

Keywords: crystal structure; Schiff base; hydrogen bonding; Hirshfeld surface analysis.

CCDC reference: 1587252

Supporting information: this article has supporting information at journals.iucr.org/e

\section{Crystal structure, Hirshfeld surface analysis and frontier molecular orbital analysis of $(E)$-4-bromo- $N^{\prime}$-(2,3-dichlorobenzylidene)benzohydrazide}

\author{
Palaniyappan Sivajeyanthi, Muthaiah Jeevaraj, Bellarmin Edison and Kasthuri \\ Balasubramani*
}

Department of Chemistry, Government Arts College (Autonomous), Thanthonimalai, Karur- 639005 , Tamil Nadu, India.
*Correspondence e-mail: manavaibala@gmail.com

The title Schiff base compound, $\mathrm{C}_{14} \mathrm{H}_{9} \mathrm{BrCl}_{2} \mathrm{~N}_{2} \mathrm{O}$, displays a trans or $E$ configuration with respect to the $\mathrm{C}=\mathrm{N}$ bond, with a dihedral angle $15.7(2)^{\circ}$ formed between the benzene rings. In the crystal, molecules are linked by $\mathrm{N}-$ $\mathrm{H} \cdots \mathrm{O}$ and $\mathrm{C}-\mathrm{H} \cdots \mathrm{O}$ hydrogen bonds, forming chains along [001] which enclose $R_{2}^{1}(6)$ loops. The intermolecular interactions were investigated by Hirshfeld surfaces analysis and two-dimensional fingerprint plots. The DFTB3LYP/6-311 G++(d,p) method was used to determine the HOMO-LUMO energy levels.

\section{Chemical context}

Schiff bases are nitrogen-containing compounds that were first obtained by the condensation reactions of aromatic amines and aldehydes (Schiff, 1864). A wide range of these compounds, with the general formula $R \mathrm{HC}=\mathrm{N} R 1$ ( $R$ and $R 1$ can be alkyl, aryl, cycloalkyl or heterocyclic groups) have been synthesized. They are of great importance in the field of coordination chemistry as they are able to form stable complexes with many metal ions (Souza et al., 1985). The chemical and biological significance of Schiff bases can be attributed to the presence of a lone electron pair in the $s p^{2}$ hybridized orbital of the nitrogen atom of the azomethine group (Singh et al., 1975). These compounds are used in the fields of organic synthesis, chemical catalysis, medicine and pharmacy as well as other new technologies (Tanaka et al., 2010). Schiff bases are also used as probes in investigating the structure of DNA (Tiwari et al., 2011) and have gained special attention in pharmacophore research and in the development of several bioactive lead molecules (Muralisankar et al., 2016). They also exhibit photochromic and thermochromic properties and have been used in information storage, electronic display systems, optical switching devices, and ophthalmic glasses (Amimoto \& Kawato, 2005). Herein, we report on the crystal structure, the Hirshfeld surface analysis and the molecular orbital analysis of the title compound, $(E)$-4-bromo- $N^{\prime}$ (2,3-dichlorobenzylidene)benzohydrazide.<smiles>O=C(N/N=C/c1cccc(Cl)c1Cl)c1ccc(Br)cc1</smiles> 
Table 1

Hydrogen-bond geometry $\left(\AA,^{\circ}\right)$.

\begin{tabular}{lllll}
\hline$D-\mathrm{H} \cdots A$ & $D-\mathrm{H}$ & $\mathrm{H} \cdots A$ & $D \cdots A$ & $D-\mathrm{H} \cdots A$ \\
\hline $\mathrm{N} 1-\mathrm{H} 1 N 1 \cdots \mathrm{O} 1^{\mathrm{i}}$ & 0.86 & 2.20 & $3.003(4)$ & 155 \\
$\mathrm{C} 8-\mathrm{H} 8 \cdots \mathrm{O} 1^{\mathrm{i}}$ & 0.93 & 2.42 & $3.234(5)$ & 146 \\
\hline
\end{tabular}

Symmetry code: (i) $x,-y+\frac{1}{2}, z+\frac{1}{2}$.

\section{Structural commentary}

The molecular structure of the title compound is illustrated in Fig. 1 . The configuration about the $\mathrm{C} 8=\mathrm{N} 2$ bond, which has a bond length of 1.271 (5) $\AA$, is $E$. The benzene rings (C1-C6 and C9-C14) are inclined to each other by 15.7 (2) ${ }^{\circ}$. The bond lengths and angles and the overall conformation of the molecule are close to those reported for a very similar compound, (E)-4-bromo- $N^{\prime}$-(2-chlorobenzylidene)benzohydrazide (Shu et al., 2009).

\section{Supramolecular features}

In the crystal, molecules are linked by $\mathrm{N}-\mathrm{H} \cdots \mathrm{O}$ and $\mathrm{C}-$ $\mathrm{H}$... O hydrogen bonds, forming chains that propagate along the [001] direction and which enclose $R_{2}^{1}(6)$ ring motifs (Fig. 2 and Table 1). Here the oxygen atom $\mathrm{O} 1$ acts as a bifurcated acceptor. There are no other significant intermolecular interactions present (see Table 2 in Hirshfeld surface analysis).

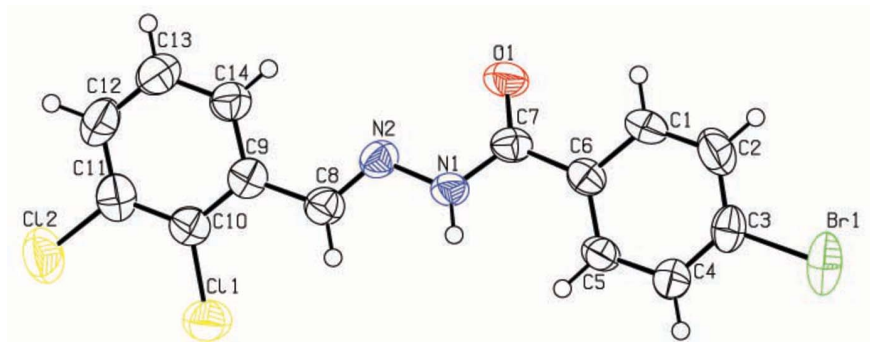

Figure 1

A view of the molecular structure of the title compound, with atom labelling. Displacement ellipsoids are drawn at the $50 \%$ probability level.

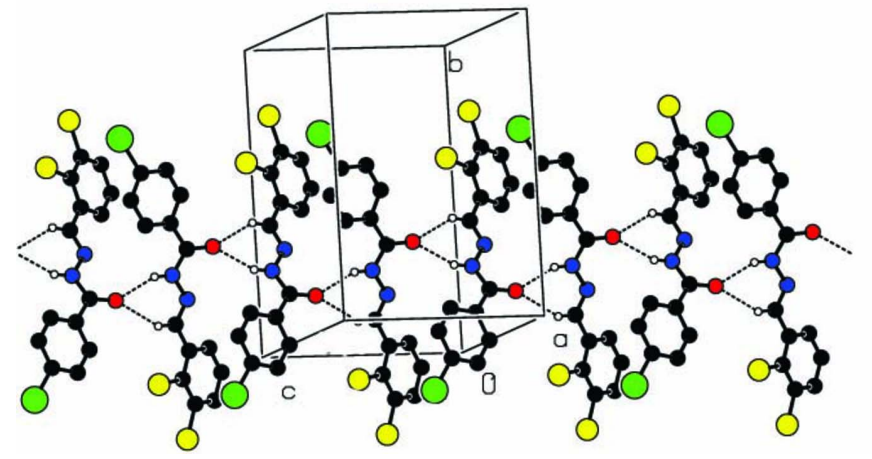

Figure 2

A partial view along the $a$ axis of the crystal packing of the title compound. Hydrogen bonds (Table 1) are shown as dashed lines, and only the $\mathrm{H}$ atoms involved in hydrogen bonding have been included.
Table 2

Intermolecular contacts $(\AA)$ for the title compound.

\begin{tabular}{llll}
\hline Atom $1 \cdots$ Atom2 & Length & Length - vdW radii & Symm. op. 2 \\
\hline $\mathrm{H} 1 N 1 \cdots \mathrm{H} 5$ & 2.136 & -0.264 & $x, y, z$ \\
$\mathrm{O} 1 \cdots \mathrm{H} 1 N 1$ & 2.200 & -0.520 & $x, \frac{1}{2}-y, z-\frac{1}{2}$ \\
$\mathrm{H} 1 N 1 \cdots \mathrm{H} 8$ & 2.242 & -0.158 & $x, y, z$ \\
$\mathrm{O} 1 \cdots \mathrm{H} 8$ & 2.421 & -0.299 & $x, \frac{1}{2}-y, z-\frac{1}{2}$ \\
$\mathrm{O} 1 \cdots \mathrm{H} 1$ & 2.520 & -0.200 & $x, y, z$ \\
$\mathrm{~N} 2 \cdots \mathrm{H} 14$ & 2.523 & -0.227 & $x, y, z$ \\
$\mathrm{H} 1 N 1 \cdots \mathrm{C} 5$ & 2.608 & -0.292 & $x, y, z$ \\
$\mathrm{~N} 1 \cdots \mathrm{H} 5$ & 2.652 & -0.098 & $x, y, z$ \\
$\mathrm{C} 1 \cdots \mathrm{H} 8$ & 2.733 & -0.217 & $x, y, z$ \\
$\mathrm{H} 1 \cdots \mathrm{Cl} 1$ & 2.931 & -0.019 & $x, \frac{1}{2}-y, z-\frac{1}{2}$ \\
$\mathrm{O} 1 \cdots \mathrm{N} 1$ & $3.003(4)$ & -0.067 & $x, \frac{1}{2}-y, z-\frac{1}{2}$ \\
$\mathrm{H} 12 \cdots \mathrm{Cl} 2$ & 3.024 & 0.074 & $x, \frac{1}{2}-y, z-\frac{1}{2}$ \\
$\mathrm{O} 1 \cdots \mathrm{C} 8$ & $3.234(5)$ & 0.014 & $x, \frac{1}{2}-y, z-\frac{1}{2}$ \\
$\mathrm{~N} 2 \cdots \mathrm{C} 5$ & $3.262(5)$ & 0.012 & $x, \frac{3}{2}-y, z-\frac{1}{2}$ \\
$\mathrm{C} 12 \cdots \mathrm{Cl} 2$ & $3.440(5)$ & -0.010 & $x, \frac{1}{2}-y, z-\frac{1}{2}$ \\
$\mathrm{C} 9 \cdots \mathrm{C} 4$ & $3.468(6)$ & 0.068 & $-x, 1-y,-z$ \\
$\mathrm{C} 8 \cdots \mathrm{C} 12$ & $3.475(5)$ & 0.075 &
\end{tabular}

\section{Hirshfeld surface analysis}

Crystal Explorer (Wolff et al., 2012) was used to generate the Hirshfeld surface and two-dimensional fingerprint plots (Rohl et al., 2008). The three-dimensional $d_{\text {norm }}$ surface is a useful tool for analysing and visualizing the intermolecular interactions, which are given in Table 2 . The $d_{\text {norm }}$ values are negative or positive depending on whether the intermolecular contact is shorter or longer than the sum of the van der Waals radii (Spackman \& Jayatilaka, 2009; McKinnon et al., 2007). The total $d_{\text {norm }}$ surface of the title compound is shown in Fig. 3. The red spots correspond to the $\mathrm{N}-\mathrm{H} \cdots \mathrm{O}$ and $\mathrm{C}-\mathrm{H} \cdots \mathrm{O}$ interactions, the most significant interactions in the crystal (Tables 1 and 2).

The two-dimensional fingerprint plots from the Hirshfeld surface analysis are shown in Fig. 4. They indicate the percentage contributions of the various intermolecular contacts to the Hirshfeld surface, the most significant are $\mathrm{Cl} \cdots \mathrm{H} / \mathrm{H} \cdots \mathrm{Cl} \quad(22.5 \%), \quad \mathrm{H} \cdots \mathrm{H} \quad(15.7 \%), \quad \mathrm{C} \cdots \mathrm{H} / \mathrm{H} \cdots \mathrm{C}$ $(13.2 \%), \mathrm{Br} \cdots \mathrm{H} / \mathrm{H} \cdots \mathrm{Br}(11.5 \%), \mathrm{C} \cdots \mathrm{C}(9.8 \%), \mathrm{O} \cdots \mathrm{H} /$ $\mathrm{H} \cdots \mathrm{O}(9.0 \%), \mathrm{N} \cdots \mathrm{H} / \mathrm{H} \cdots \mathrm{N}(4.9 \%)$, and $\mathrm{Br} \cdots \mathrm{Cl} / \mathrm{Cl} \cdots \mathrm{Br}$ $(3.3 \%)$, as shown in Fig. 4 , cf Table 2.

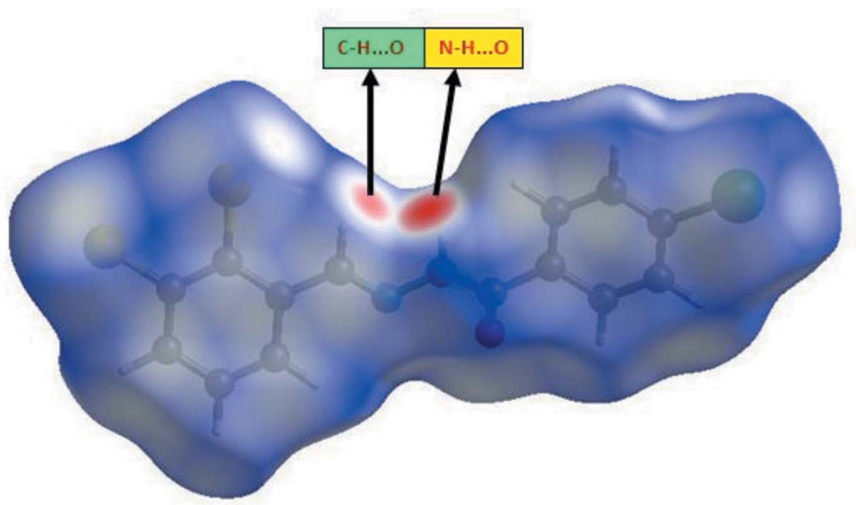

Figure 3

Hirshfeld surface mapped over $d_{\text {norm }}$ for the title compound. [add range of dnorm to legend] 


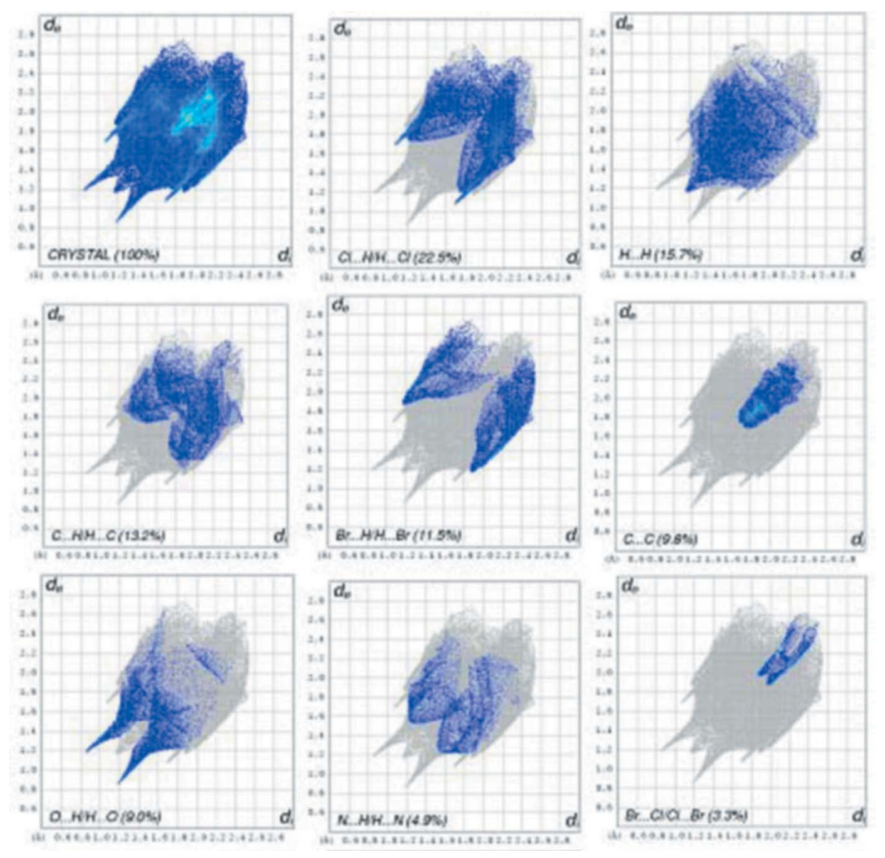

Figure 4

Two-dimensional fingerprint plots of the crystal with the relative contributions of the atom pairs to the Hirshfeld surface.

\section{Frontier molecular orbital calculations}

The HOMO (highest occupied molecular orbital) acts as an electron donor and the LUMO (lowest occupied molecular orbital) as an electron acceptor. If the energy gap is small then the molecule is highly polarizable and has high chemical reactivity. The energy levels of the title compound were computed using the DFT-B3LYP/6-311G++(d,p) method (Sivajeyanthi et al., 2017). The energy gap between HOMOLUMO orbitals, which determines the chemical stability, chemical hardness, chemical potential, electronegativity and the electrophilicity index are shown in Fig. 5 and details are given in Table 3. The frontier molecular orbital LUMO is located over the whole of the molecule. The energy gap of the molecule clearly shows the charge-transfer interaction involving donor and acceptor groups. The chemical hardness and softness of a molecule is a sign of its chemical stability. From the HOMO-LUMO energy gap, we can see whether or not the molecule is hard or soft. If the energy gap is large, the molecule is hard and if small the molecule is soft. Soft molecules are more polarizable than hard ones because they need less energy for excitation. From the data presented in Table 3, we conclude that the energy gap is large, hence the title molecule is a hard material and will be difficult to polarize.

\section{Database survey}

A search of the Cambridge Structural Database (CSD, version 5.39, last update August 2018; Groom et al., 2016) for 4-bromo(benzylidene)benzohydrazides yielded six structures. They include the following analogues: 2,4-dihydroxybenzylidene [ATOSEJ (Mohanraj et al., 2016) and ATOSEJ01 (Arunagiri
Table 3

Calculated frontier molecular orbital analysis of the title compound.

\begin{tabular}{lr}
\hline EHOMO & $-6.7318 \mathrm{eV}$ \\
ELUMO & $-2.4441 \mathrm{eV}$ \\
EHOMO-1 & $-7.2556 \mathrm{eV}$ \\
ELUMO+1 & $-1.6506 \mathrm{eV}$ \\
EHOMO-ELUMO gap & $4.2877 \mathrm{eV}$ \\
EHOMO-1 ELUMO+1 gap & $5.6050 \mathrm{eV}$ \\
Chemical hardness $(\eta)$ & $2.1438 \mathrm{eV}$ \\
Chemical potential $(\mu)$ & $4.5879 \mathrm{eV}$ \\
Electronegativity $(\chi)$ & $-4.5879 \mathrm{eV}$ \\
Electrophilicity index $(\omega)$ & $4.9092 \mathrm{eV}$ \\
\hline
\end{tabular}

et al., 2018)], 2-nitrobenzylidene (EGUSEF; Zhang et al., 2009), 2-chlorobenzylidene (HOTDAW; Shu et al., 2009), 2hydroxy-1-naphthylmethylene (IFUSEI; Diao et al., 2008), 2hydroxy-5-methoxybenzylidene (OBUBUL; Wang et al., 2017) and

4-hydroxy-3-methoxybenzylidene (YAWXOL; Horkaew et al., 2012). They all have an $E$ configuration about the $\mathrm{C}=\mathrm{N}$ bond. The $\mathrm{N}-\mathrm{N}$ bond lengths vary from 1.366 (4) to $1.396(5) \AA$ while the $\mathrm{C}=\mathrm{N}$ bond lengths vary from 1.264 (4) to 1.285 (2) $\AA$. The values observed for the title compound, respectively, 1.391 (4) and 1.271 (5) A, fall within these limits. The dihedral angle between the two benzene rings varies from as little as $4.12(17)^{\circ}$ in EGUSEF to $49.08(18)^{\circ}$ in ATOSEJ01. In the title compound this dihedral angle is $15.7(2)^{\circ}$, similar to the values observed for HOTDAW, the 2-chlorobenzylidene analogue, and for YAWXOL, the 4-hydroxy-3-methoxybenzylidene analogue, for which the dihedral angles are 11.43 (16) and $13.92(6)^{\circ}$, respectively.

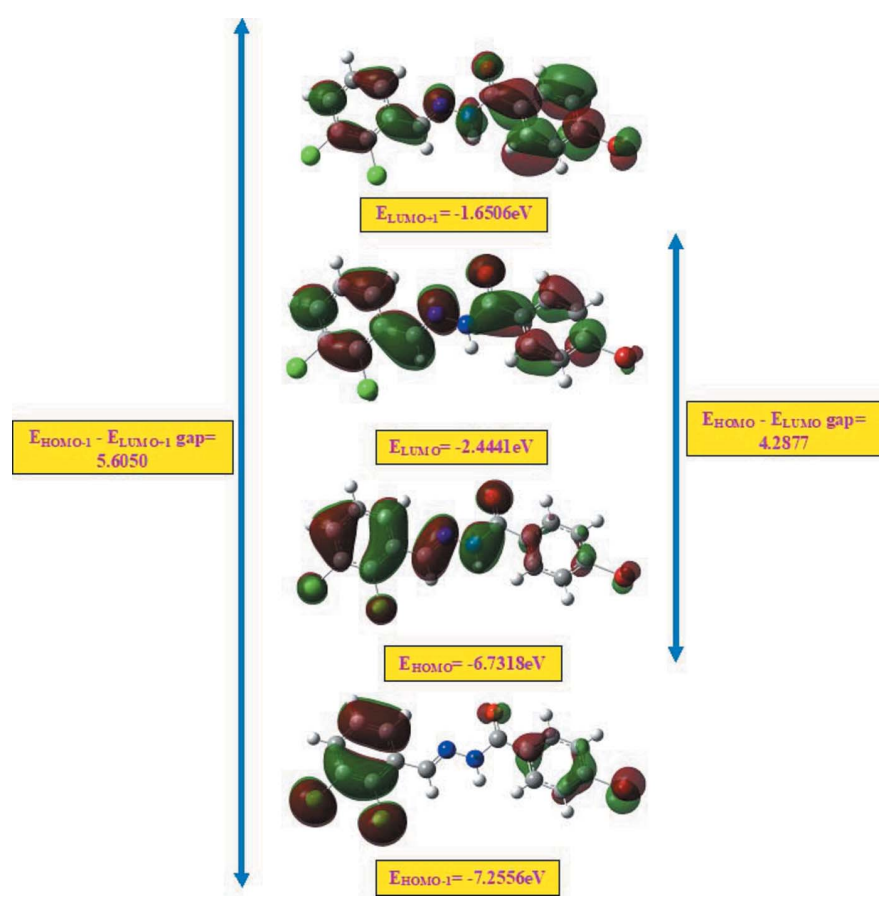

Figure 5

Molecular orbital energy levels of the title compound. 


\section{Synthesis and crystallization}

The title compound was synthesized by the reaction of 1:1 molar ratio mixture of a hot ethanolic solution $(20 \mathrm{ml})$ of 4-bromobenzohydrazide $(0.213 \mathrm{mg}$, Aldrich) and 2,3-dichlorobenzaldehyde $(0.175 \mathrm{mg}$, Aldrich), which was refluxed for $8 \mathrm{~h}$. The solution was then cooled and kept at room temperature. The powder obtained was recrystallized from dimethyl sulfoxide (DMSO). Colourless block-like crystals suitable for the X-ray diffraction analysis were obtained in a few days.

\section{Refinement}

Crystal data, data collection and structure refinement details are summarized in Table 4 . The hydrogen atoms were positioned geometrically and refined using a riding model: $\mathrm{C}-\mathrm{H}=$ $0.93 \AA, \mathrm{N}-\mathrm{H}=0.86 \AA$, with $U_{\text {iso }}(\mathrm{H})=1.2 U_{\text {eq }}(\mathrm{N}, \mathrm{C})$.

\section{Funding information}

KB and PS thank the Department of Science and Technology (DST-SERB), grant No. SB/FT/CS-058/2013, New Delhi, India, for financial support.

\section{References}

Altomare, A., Cascarano, G., Giacovazzo, C. \& Guagliardi, A. (1993). J. Appl. Cryst. 26, 343-350.

Amimoto, K. \& Kawato, T. (2005). J. Photochem. Photobiol. Photochem. Rev. 6, 207-226.

Arunagiri, C., Anitha, A. G., Subashini, A. \& Selvakumar, S. (2018). J. Mol. Struct. 1163, 368-378.

Bruker (2004). SAINT, APEX2, XPREP and $S A D A B S$. Bruker AXS Inc., Madison, Wisconsin, USA.

Diao, Y.-P., Zhang, Q.-H., Wang, D.-C. \& Deng, X.-M. (2008). Acta Cryst. E64, o2070.

Groom, C. R., Bruno, I. J., Lightfoot, M. P. \& Ward, S. C. (2016). Acta Cryst. B72, 171-179.

Horkaew, J., Chantrapromma, S., Anantapong, T., Kanjana-Opas, A. \& Fun, H.-K. (2012). Acta Cryst. E68, o1069-o1070.

McKinnon, J. J., Jayatilaka, D. \& Spackman, M. A. (2007). Chem. Commun. 3814-3816.

Mohanraj, M., Ayyannan, G., Raja, G. \& Jayabalakrishnan, C. (2016). Mater. Sci. Eng. C, 69, 1297-1306.

Muralisankar, M., Haribabu, J., Bhuvanesh, N. S. P., Karvembu, R. \& Sreekanth, A. (2016). Inorg. Chim. Acta, 449, 82-95.

Rohl, A. L., Moret, M., Kaminsky, W., Claborn, K., McKinnon, J. J. \& Kahr, B. (2008). Cryst. Growth Des. 8, 4517-4525.

Schiff, H. (1864). Justus Liebigs Ann. Chem. 131, 118-119.

Sheldrick, G. M. (2015). Acta Cryst. C71, 3-8.
Table 4

Experimental details.

\begin{tabular}{|c|c|}
\hline \multicolumn{2}{|l|}{ Crystal data } \\
\hline Chemical formula & $\mathrm{C}_{14} \mathrm{H}_{9} \mathrm{BrCl}_{2} \mathrm{~N}_{2} \mathrm{O}$ \\
\hline$M_{\mathrm{r}}$ & 372.04 \\
\hline Crystal system, space group & Monoclinic, $P 2_{1} / c$ \\
\hline Temperature $(\mathrm{K})$ & 296 \\
\hline$a, b, c(\AA)$ & $\begin{array}{l}11.1952(18), 14.055(2), \\
9.3050(12)\end{array}$ \\
\hline$\beta\left(^{\circ}\right)$ & $96.446(6)$ \\
\hline$V\left(\AA^{3}\right)$ & $1454.8(4)$ \\
\hline$Z$ & 4 \\
\hline Radiation type & Mo $K \alpha$ \\
\hline$\mu\left(\mathrm{mm}^{-1}\right)$ & 3.19 \\
\hline Crystal size $(\mathrm{mm})$ & $0.30 \times 0.20 \times 0.20$ \\
\hline \multicolumn{2}{|l|}{ Data collection } \\
\hline Diffractometer & Bruker Kappa APEXII CCD \\
\hline Absorption correction & $\begin{array}{l}\text { Multi-scan (SADABS; Bruker, } \\
\text { 2004) }\end{array}$ \\
\hline$T_{\min }, T_{\max }$ & $0.448,0.568$ \\
\hline $\begin{array}{l}\text { No. of measured, independent and } \\
\text { observed }[I>2 \sigma(I)] \text { reflections }\end{array}$ & $11392,3363,1724$ \\
\hline$R_{\text {int }}$ & 0.050 \\
\hline$(\sin \theta / \lambda)_{\max }\left(\AA^{-1}\right)$ & 0.666 \\
\hline \multicolumn{2}{|l|}{ Refinement } \\
\hline$R\left[F^{2}>2 \sigma\left(F^{2}\right)\right], w R\left(F^{2}\right), S$ & $0.051,0.173,0.94$ \\
\hline No. of reflections & 3363 \\
\hline No. of parameters & 181 \\
\hline $\mathrm{H}$-atom treatment & $\mathrm{H}$-atom parameters constrained \\
\hline$\Delta \rho_{\max }, \Delta \rho_{\min }\left(\mathrm{e} \AA^{-3}\right)$ & $0.70,-0.63$ \\
\hline
\end{tabular}

Computer programs: APEX2, SAINT and XPREP (Bruker, 2004), SIR92 (Altomare et al., 1993), SHELXL2017 (Sheldrick, 2015) and PLATON (Spek, 2009).

Shu, X.-H., Diao, Y.-P., Li, M.-L., Yan, X. \& Liu, J. (2009). Acta Cryst. E65, o1034.

Singh, P., Goel, R. L. \& Singh, B. P. (1975). J. Indian Chem. Soc. 52, 958-959.

Sivajeyanthi, P., Jeevaraj, M., Balasubramani, K., Viswanathan, V. \& Velmurugan, D. (2017). Chem. Data Collect. 11-12, 220-231.

Souza, P., Garcia-Vazquez, J. A. \& Masaguer, J. R. (1985). Transition Met. Chem. 10, 410-412.

Spackman, M. A. \& Jayatilaka, D. (2009). CrystEngComm, 11, 19-32. Spek, A. L. (2009). Acta Cryst. D65, 148-155.

Tanaka, K., Shimoura, R. \& Caira, M. R. (2010). Tetrahedron Lett. 51, 449-452.

Tiwari, A. D., Mishra, A. K., Mishra, B. B., Mamba, B. B., Maji, B. \& Bhattacharya, S. (2011). Spectrochim. Acta A, 79, 1050-1056.

Wang, J., Qu, D., Lei, J.-X. \& You, Z. (2017). J. Coord. Chem. 70, 544555 .

Wolff, S. K., Grimwood, D. J., McKinnon, J. J., Turner, M. J., Jayatilaka, D. \& Spackman, M. A. (2012). Crystal Explorer. University of Western Australia, Australia.

Zhang, M.-J., Yin, L.-Z., Wang, D.-C., Deng, X.-M. \& Liu, J.-B. (2009). Acta Cryst. E65, o508. 


\section{supporting information}

Acta Cryst. (2019). E75, 324-327 [https://doi.org/10.1107/S2056989019001816]

\section{Crystal structure, Hirshfeld surface analysis and frontier molecular orbital} analysis of (E)-4-bromo- $N$ '-(2,3-dichlorobenzylidene)benzohydrazide

\section{Palaniyappan Sivajeyanthi, Muthaiah Jeevaraj, Bellarmin Edison and Kasthuri Balasubramani}

Computing details

Data collection: APEX2 (Bruker, 2004); cell refinement: APEX2 and SAINT (Bruker, 2004); data reduction: SAINT and XPREP (Bruker, 2004); program(s) used to solve structure: SIR92 (Altomare et al., 1993); program(s) used to refine structure: SHELXL2017 (Sheldrick, 2015); molecular graphics: PLATON (Spek, 2009); software used to prepare material for publication: SHELXL2017 (Sheldrick, 2015) and PLATON (Spek, 2009).

(E)-4-Bromo- $N^{\prime}$-(2,3-dichlorobenzylidene)benzohydrazide

Crystal data

$\mathrm{C}_{14} \mathrm{H}_{9} \mathrm{BrCl}_{2} \mathrm{~N}_{2} \mathrm{O}$

$M_{r}=372.04$

Monoclinic, $P 2{ }_{1} / C$

$a=11.1952(18) \AA$

$b=14.055(2) \AA$

$c=9.3050(12) \AA$

$\beta=96.446(6)^{\circ}$

$V=1454.8(4) \AA^{3}$

$Z=4$

\section{Data collection}

Bruker Kappa APEXII CCD diffractometer

Radiation source: fine-focus sealed tube $\omega$ and $\varphi$ scan

Absorption correction: multi-scan

(SADABS; Bruker, 2004)

$T_{\min }=0.448, T_{\max }=0.568$

11392 measured reflections

\section{Refinement}

Refinement on $F^{2}$

Least-squares matrix: full

$R\left[F^{2}>2 \sigma\left(F^{2}\right)\right]=0.051$

$w R\left(F^{2}\right)=0.173$

$S=0.94$

3363 reflections

181 parameters

0 restraints

Primary atom site location: structure-invariant direct methods
$F(000)=736$

$D_{\mathrm{x}}=1.699 \mathrm{Mg} \mathrm{m}^{-3}$

Mo $K \alpha$ radiation, $\lambda=0.71073 \AA$

Cell parameters from 3186 reflections

$\theta=4.7-47.5^{\circ}$

$\mu=3.19 \mathrm{~mm}^{-1}$

$T=296 \mathrm{~K}$

Block, colourless

$0.30 \times 0.20 \times 0.20 \mathrm{~mm}$

3363 independent reflections

1724 reflections with $I>2 \sigma(I)$

$R_{\text {int }}=0.050$

$\theta_{\text {max }}=28.3^{\circ}, \theta_{\text {min }}=2.6^{\circ}$

$h=-14 \rightarrow 14$

$k=-18 \rightarrow 18$

$l=-8 \rightarrow 12$

Secondary atom site location: difference Fourier map

Hydrogen site location: inferred from neighbouring sites

$\mathrm{H}$-atom parameters constrained

$w=1 /\left[\sigma^{2}\left(F_{\mathrm{o}}{ }^{2}\right)+(0.1 P)^{2}\right]$

where $P=\left(F_{\mathrm{o}}{ }^{2}+2 F_{\mathrm{c}}{ }^{2}\right) / 3$

$(\Delta / \sigma)_{\max }<0.001$

$\Delta \rho_{\max }=0.70 \mathrm{e} \AA^{-3}$

$\Delta \rho_{\min }=-0.63$ e $\AA^{-3}$ 


\section{Special details}

Geometry. All esds (except the esd in the dihedral angle between two 1.s. planes) are estimated using the full covariance matrix. The cell esds are taken into account individually in the estimation of esds in distances, angles and torsion angles; correlations between esds in cell parameters are only used when they are defined by crystal symmetry. An approximate (isotropic) treatment of cell esds is used for estimating esds involving l.s. planes.

Refinement. Refinement of $\mathrm{F}^{2}$ against ALL reflections. The weighted R-factor $\mathrm{wR}$ and goodness of fit $\mathrm{S}$ are based on $\mathrm{F}^{2}$, conventional $\mathrm{R}$-factors $\mathrm{R}$ are based on $\mathrm{F}$, with $\mathrm{F}$ set to zero for negative $\mathrm{F}^{2}$. The threshold expression of $\mathrm{F}^{2}>2 \operatorname{sigma}\left(\mathrm{F}^{2}\right)$ is used only for calculating R-factors(gt) etc. and is not relevant to the choice of reflections for refinement. R-factors based on $\mathrm{F}^{2}$ are statistically about twice as large as those based on F, and R- factors based on ALL data will be even larger.

Fractional atomic coordinates and isotropic or equivalent isotropic displacement parameters $\left(\hat{A}^{2}\right)$

\begin{tabular}{lllll}
\hline & $x$ & $y$ & $z$ & $U_{\text {iso }} / U_{\text {eq }}$ \\
\hline Br1 & $0.45376(6)$ & $-0.17154(4)$ & $0.33892(7)$ & $0.0891(3)$ \\
C11 & $0.24263(13)$ & $0.60728(9)$ & $0.12531(12)$ & $0.0749(4)$ \\
C12 & $0.11288(14)$ & $0.77291(9)$ & $-0.05544(15)$ & $0.0843(5)$ \\
O1 & $0.2713(3)$ & $0.1659(2)$ & $-0.1706(3)$ & $0.0628(9)$ \\
N1 & $0.2462(3)$ & $0.2532(2)$ & $0.0288(3)$ & $0.0456(9)$ \\
H1N1 & 0.253851 & 0.257938 & 0.121571 & $0.055^{*}$ \\
N2 & $0.1988(3)$ & $0.3275(2)$ & $-0.0585(4)$ & $0.0478(9)$ \\
C6 & $0.3268(4)$ & $0.0931(3)$ & $0.0576(4)$ & $0.0439(10)$ \\
C9 & $0.1358(4)$ & $0.4878(3)$ & $-0.0799(4)$ & $0.0470(10)$ \\
C7 & $0.2801(4)$ & $0.1731(3)$ & $-0.0370(4)$ & $0.0452(10)$ \\
C5 & $0.3717(4)$ & $0.1033(3)$ & $0.2017(4)$ & $0.0473(10)$ \\
H5 & 0.375456 & 0.163419 & 0.243713 & $0.057^{*}$ \\
C10 & $0.1524(4)$ & $0.5818(3)$ & $-0.0321(4)$ & $0.0483(10)$ \\
C3 & $0.4067(4)$ & $-0.0633(3)$ & $0.2236(5)$ & $0.0527(11)$ \\
C4 & $0.4112(4)$ & $0.0249(3)$ & $0.2838(5)$ & $0.0539(11)$ \\
H4 & 0.440892 & 0.032600 & 0.380460 & $0.065^{*}$ \\
C8 & $0.1896(4)$ & $0.4073(3)$ & $0.0037(4)$ & $0.0496(11)$ \\
H8 & 0.216763 & 0.414398 & 0.101258 & $0.060^{*}$ \\
C11 & $0.0950(5)$ & $0.6564(3)$ & $-0.1127(5)$ & $0.0569(12)$ \\
C12 & $0.0226(5)$ & $0.6387(4)$ & $-0.2402(5)$ & $0.0663(14)$ \\
H12 & -0.015996 & 0.688339 & -0.292702 & $0.080^{*}$ \\
C14 & $0.0639(4)$ & $0.4716(3)$ & $-0.2103(5)$ & $0.0555(12)$ \\
H14 & 0.053241 & 0.409731 & -0.244882 & $0.067^{*}$ \\
C1 & $0.3261(5)$ & $0.0017(3)$ & $-0.0023(5)$ & $0.0636(13)$ \\
H1 & 0.299196 & -0.006402 & -0.099732 & $0.076^{*}$ \\
C13 & $0.0084(5)$ & $0.5456(4)$ & $-0.2885(5)$ & $0.0674(14)$ \\
H13 & -0.039161 & 0.533003 & -0.374844 & $0.081^{*}$ \\
C2 & $0.3643(5)$ & $-0.0761(3)$ & $0.0792(6)$ & $0.0716(15)$ \\
H2 & 0.361649 & -0.136545 & 0.038188 & $0.086^{*}$ \\
& & & &
\end{tabular}

Atomic displacement parameters $\left(\AA^{2}\right)$

\begin{tabular}{lllllll}
\hline & $U^{11}$ & $U^{22}$ & $U^{33}$ & $U^{12}$ & $U^{13}$ & $U^{23}$ \\
\hline Br1 & $0.0922(5)$ & $0.0604(4)$ & $0.1158(6)$ & $0.0313(3)$ & $0.0164(4)$ & $0.0248(3)$ \\
C11 & $0.1014(11)$ & $0.0608(7)$ & $0.0589(8)$ & $-0.0126(7)$ & $-0.0069(7)$ & $0.0007(6)$ \\
C12 & $0.1127(13)$ & $0.0482(7)$ & $0.0933(10)$ & $0.0101(7)$ & $0.0175(9)$ & $0.0065(6)$
\end{tabular}


supporting information

\begin{tabular}{lllllll} 
O1 & $0.094(3)$ & $0.0564(19)$ & $0.0371(17)$ & $-0.0015(16)$ & $0.0027(16)$ & $-0.0063(13)$ \\
N1 & $0.062(2)$ & $0.0421(19)$ & $0.0319(17)$ & $-0.0021(17)$ & $0.0032(16)$ & $0.0005(14)$ \\
N2 & $0.052(2)$ & $0.047(2)$ & $0.0430(19)$ & $-0.0024(17)$ & $0.0010(17)$ & $0.0065(16)$ \\
C6 & $0.042(3)$ & $0.042(2)$ & $0.048(2)$ & $-0.0064(19)$ & $0.0082(19)$ & $-0.0053(18)$ \\
C9 & $0.044(3)$ & $0.052(2)$ & $0.047(2)$ & $-0.001(2)$ & $0.012(2)$ & $0.0049(19)$ \\
C7 & $0.049(3)$ & $0.046(2)$ & $0.041(2)$ & $-0.0128(19)$ & $0.004(2)$ & $-0.0007(19)$ \\
C5 & $0.060(3)$ & $0.040(2)$ & $0.041(2)$ & $-0.003(2)$ & $0.005(2)$ & $-0.0070(17)$ \\
C10 & $0.050(3)$ & $0.052(2)$ & $0.044(2)$ & $-0.003(2)$ & $0.013(2)$ & $0.0009(18)$ \\
C3 & $0.041(3)$ & $0.052(2)$ & $0.066(3)$ & $0.013(2)$ & $0.013(2)$ & $0.008(2)$ \\
C4 & $0.061(3)$ & $0.050(2)$ & $0.050(2)$ & $0.009(2)$ & $0.004(2)$ & $0.002(2)$ \\
C8 & $0.059(3)$ & $0.047(2)$ & $0.043(2)$ & $-0.004(2)$ & $0.007(2)$ & $0.0023(19)$ \\
C11 & $0.062(3)$ & $0.053(3)$ & $0.060(3)$ & $0.003(2)$ & $0.025(3)$ & $0.010(2)$ \\
C12 & $0.068(4)$ & $0.069(3)$ & $0.062(3)$ & $0.017(3)$ & $0.005(3)$ & $0.019(3)$ \\
C14 & $0.058(3)$ & $0.061(3)$ & $0.047(3)$ & $0.006(2)$ & $0.004(2)$ & $-0.002(2)$ \\
C1 & $0.085(4)$ & $0.051(3)$ & $0.051(3)$ & $0.000(3)$ & $-0.008(2)$ & $-0.018(2)$ \\
C13 & $0.069(4)$ & $0.078(4)$ & $0.053(3)$ & $0.012(3)$ & $-0.004(3)$ & $0.002(3)$ \\
C2 & $0.076(4)$ & $0.043(3)$ & $0.093(4)$ & $0.006(2)$ & $-0.004(3)$ & $-0.017(3)$ \\
& & & & & & \\
\hline
\end{tabular}

Geometric parameters $\left(A,{ }^{\circ}\right)$

\begin{tabular}{|c|c|c|c|}
\hline $\mathrm{Br} 1-\mathrm{C} 3$ & $1.901(4)$ & $\mathrm{C} 5-\mathrm{H} 5$ & 0.9300 \\
\hline $\mathrm{C} 11-\mathrm{C} 10$ & $1.721(4)$ & $\mathrm{C} 10-\mathrm{C} 11$ & $1.402(6)$ \\
\hline $\mathrm{Cl} 2-\mathrm{C} 11$ & $1.727(5)$ & $\mathrm{C} 3-\mathrm{C} 4$ & $1.359(6)$ \\
\hline $\mathrm{O} 1-\mathrm{C} 7$ & $1.240(5)$ & $\mathrm{C} 3-\mathrm{C} 2$ & $1.385(6)$ \\
\hline $\mathrm{N} 1-\mathrm{C} 7$ & $1.356(5)$ & $\mathrm{C} 4-\mathrm{H} 4$ & 0.9300 \\
\hline $\mathrm{N} 1-\mathrm{N} 2$ & $1.391(4)$ & $\mathrm{C} 8-\mathrm{H} 8$ & 0.9300 \\
\hline N1-H1N1 & 0.8600 & $\mathrm{C} 11-\mathrm{C} 12$ & $1.383(7)$ \\
\hline $\mathrm{N} 2-\mathrm{C} 8$ & $1.271(5)$ & $\mathrm{C} 12-\mathrm{C} 13$ & $1.386(7)$ \\
\hline $\mathrm{C} 6-\mathrm{C} 5$ & $1.385(5)$ & $\mathrm{C} 12-\mathrm{H} 12$ & 0.9300 \\
\hline $\mathrm{C} 6-\mathrm{C} 1$ & $1.400(5)$ & $\mathrm{C} 14-\mathrm{C} 13$ & $1.377(6)$ \\
\hline C6- 77 & $1.486(6)$ & C14-H14 & 0.9300 \\
\hline $\mathrm{C} 9-\mathrm{C} 14$ & $1.398(6)$ & $\mathrm{C} 1-\mathrm{C} 2$ & $1.372(7)$ \\
\hline $\mathrm{C} 9-\mathrm{C} 10$ & $1.400(6)$ & $\mathrm{C} 1-\mathrm{H} 1$ & 0.9300 \\
\hline $\mathrm{C} 9-\mathrm{C} 8$ & $1.464(6)$ & $\mathrm{C} 13-\mathrm{H} 13$ & 0.9300 \\
\hline $\mathrm{C} 5-\mathrm{C} 4$ & $1.385(6)$ & $\mathrm{C} 2-\mathrm{H} 2$ & 0.9300 \\
\hline $\mathrm{C} 7-\mathrm{N} 1-\mathrm{N} 2$ & $117.8(3)$ & $\mathrm{C} 3-\mathrm{C} 4-\mathrm{H} 4$ & 119.9 \\
\hline $\mathrm{C} 7-\mathrm{N} 1-\mathrm{H} 1 \mathrm{~N} 1$ & 121.1 & $\mathrm{C} 5-\mathrm{C} 4-\mathrm{H} 4$ & 119.9 \\
\hline $\mathrm{N} 2-\mathrm{N} 1-\mathrm{H} 1 \mathrm{~N} 1$ & 121.1 & $\mathrm{~N} 2-\mathrm{C} 8-\mathrm{C} 9$ & $119.3(4)$ \\
\hline $\mathrm{C} 8-\mathrm{N} 2-\mathrm{N} 1$ & $116.2(3)$ & $\mathrm{N} 2-\mathrm{C} 8-\mathrm{H} 8$ & 120.3 \\
\hline $\mathrm{C} 5-\mathrm{C} 6-\mathrm{C} 1$ & $117.7(4)$ & $\mathrm{C} 9-\mathrm{C} 8-\mathrm{H} 8$ & 120.3 \\
\hline $\mathrm{C} 5-\mathrm{C} 6-\mathrm{C} 7$ & $124.1(4)$ & $\mathrm{C} 12-\mathrm{C} 11-\mathrm{C} 10$ & $120.9(4)$ \\
\hline $\mathrm{C} 1-\mathrm{C} 6-\mathrm{C} 7$ & $118.2(4)$ & $\mathrm{C} 12-\mathrm{C} 11-\mathrm{Cl} 2$ & $118.2(4)$ \\
\hline $\mathrm{C} 14-\mathrm{C} 9-\mathrm{C} 10$ & $118.2(4)$ & $\mathrm{C} 10-\mathrm{C} 11-\mathrm{Cl} 2$ & $121.0(4)$ \\
\hline $\mathrm{C} 14-\mathrm{C} 9-\mathrm{C} 8$ & $119.9(4)$ & $\mathrm{C} 11-\mathrm{C} 12-\mathrm{C} 13$ & $118.9(4)$ \\
\hline $\mathrm{C} 10-\mathrm{C} 9-\mathrm{C} 8$ & $121.9(4)$ & $\mathrm{C} 11-\mathrm{C} 12-\mathrm{H} 12$ & 120.6 \\
\hline $\mathrm{O} 1-\mathrm{C} 7-\mathrm{N} 1$ & $121.7(4)$ & $\mathrm{C} 13-\mathrm{C} 12-\mathrm{H} 12$ & 120.6 \\
\hline $\mathrm{O} 1-\mathrm{C} 7-\mathrm{C} 6$ & $121.0(4)$ & $\mathrm{C} 13-\mathrm{C} 14-\mathrm{C} 9$ & $121.2(4)$ \\
\hline
\end{tabular}




\begin{tabular}{|c|c|c|c|}
\hline $\mathrm{N} 1-\mathrm{C} 7-\mathrm{C} 6$ & $117.3(3)$ & $\mathrm{C} 13-\mathrm{C} 14-\mathrm{H} 14$ & 119.4 \\
\hline $\mathrm{C} 4-\mathrm{C} 5-\mathrm{C} 6$ & $120.7(4)$ & $\mathrm{C} 9-\mathrm{C} 14-\mathrm{H} 14$ & 119.4 \\
\hline $\mathrm{C} 4-\mathrm{C} 5-\mathrm{H} 5$ & 119.7 & $\mathrm{C} 2-\mathrm{C} 1-\mathrm{C} 6$ & $121.6(4)$ \\
\hline $\mathrm{C} 6-\mathrm{C} 5-\mathrm{H} 5$ & 119.7 & $\mathrm{C} 2-\mathrm{C} 1-\mathrm{H} 1$ & 119.2 \\
\hline $\mathrm{C} 9-\mathrm{C} 10-\mathrm{C} 11$ & $120.0(4)$ & $\mathrm{C} 6-\mathrm{C} 1-\mathrm{H} 1$ & 119.2 \\
\hline $\mathrm{C} 9-\mathrm{C} 10-\mathrm{Cl} 1$ & $120.7(3)$ & $\mathrm{C} 14-\mathrm{C} 13-\mathrm{C} 12$ & $120.9(5)$ \\
\hline $\mathrm{C} 11-\mathrm{C} 10-\mathrm{C} 11$ & $119.3(3)$ & $\mathrm{C} 14-\mathrm{C} 13-\mathrm{H} 13$ & 119.6 \\
\hline $\mathrm{C} 4-\mathrm{C} 3-\mathrm{C} 2$ & $120.7(4)$ & $\mathrm{C} 12-\mathrm{C} 13-\mathrm{H} 13$ & 119.6 \\
\hline $\mathrm{C} 4-\mathrm{C} 3-\mathrm{Br} 1$ & $120.1(3)$ & $\mathrm{C} 1-\mathrm{C} 2-\mathrm{C} 3$ & $118.9(4)$ \\
\hline $\mathrm{C} 2-\mathrm{C} 3-\mathrm{Br} 1$ & $119.1(3)$ & $\mathrm{C} 1-\mathrm{C} 2-\mathrm{H} 2$ & 120.5 \\
\hline $\mathrm{C} 3-\mathrm{C} 4-\mathrm{C} 5$ & $120.3(4)$ & $\mathrm{C} 3-\mathrm{C} 2-\mathrm{H} 2$ & 120.5 \\
\hline $\mathrm{C} 7-\mathrm{N} 1-\mathrm{N} 2-\mathrm{C} 8$ & $-166.9(4)$ & $\mathrm{C} 14-\mathrm{C} 9-\mathrm{C} 8-\mathrm{N} 2$ & $19.2(6)$ \\
\hline $\mathrm{N} 2-\mathrm{N} 1-\mathrm{C} 7-\mathrm{O} 1$ & $1.1(6)$ & $\mathrm{C} 10-\mathrm{C} 9-\mathrm{C} 8-\mathrm{N} 2$ & $-162.0(4)$ \\
\hline $\mathrm{N} 2-\mathrm{N} 1-\mathrm{C} 7-\mathrm{C} 6$ & $-178.0(4)$ & $\mathrm{C} 9-\mathrm{C} 10-\mathrm{C} 11-\mathrm{C} 12$ & $-0.6(7)$ \\
\hline $\mathrm{C} 5-\mathrm{C} 6-\mathrm{C} 7-\mathrm{O} 1$ & $160.5(4)$ & $\mathrm{C} 11-\mathrm{C} 10-\mathrm{C} 11-\mathrm{C} 12$ & $179.5(4)$ \\
\hline $\mathrm{C} 1-\mathrm{C} 6-\mathrm{C} 7-\mathrm{O} 1$ & $-19.2(6)$ & $\mathrm{C} 9-\mathrm{C} 10-\mathrm{C} 11-\mathrm{Cl} 2$ & $179.5(3)$ \\
\hline $\mathrm{C} 5-\mathrm{C} 6-\mathrm{C} 7-\mathrm{N} 1$ & $-20.4(6)$ & $\mathrm{C} 11-\mathrm{C} 10-\mathrm{C} 11-\mathrm{Cl} 2$ & $-0.4(5)$ \\
\hline $\mathrm{C} 1-\mathrm{C} 6-\mathrm{C} 7-\mathrm{N} 1$ & $160.0(4)$ & $\mathrm{C} 10-\mathrm{C} 11-\mathrm{C} 12-\mathrm{C} 13$ & $-0.7(7)$ \\
\hline $\mathrm{C} 1-\mathrm{C} 6-\mathrm{C} 5-\mathrm{C} 4$ & $-1.8(6)$ & $\mathrm{Cl} 2-\mathrm{C} 11-\mathrm{C} 12-\mathrm{C} 13$ & $179.2(4)$ \\
\hline $\mathrm{C} 7-\mathrm{C} 6-\mathrm{C} 5-\mathrm{C} 4$ & $178.5(4)$ & $\mathrm{C} 10-\mathrm{C} 9-\mathrm{C} 14-\mathrm{C} 13$ & $-1.4(7)$ \\
\hline $\mathrm{C} 14-\mathrm{C} 9-\mathrm{C} 10-\mathrm{C} 11$ & $1.6(6)$ & $\mathrm{C} 8-\mathrm{C} 9-\mathrm{C} 14-\mathrm{C} 13$ & $177.5(4)$ \\
\hline $\mathrm{C} 8-\mathrm{C} 9-\mathrm{C} 10-\mathrm{C} 11$ & $-177.3(4)$ & $\mathrm{C} 5-\mathrm{C} 6-\mathrm{C} 1-\mathrm{C} 2$ & $2.4(7)$ \\
\hline $\mathrm{C} 14-\mathrm{C} 9-\mathrm{C} 10-\mathrm{Cl1}$ & $-178.5(3)$ & $\mathrm{C} 7-\mathrm{C} 6-\mathrm{C} 1-\mathrm{C} 2$ & $-177.9(4)$ \\
\hline $\mathrm{C} 8-\mathrm{C} 9-\mathrm{C} 10-\mathrm{Cl} 1$ & $2.6(6)$ & $\mathrm{C} 9-\mathrm{C} 14-\mathrm{C} 13-\mathrm{C} 12$ & $0.1(7)$ \\
\hline $\mathrm{C} 2-\mathrm{C} 3-\mathrm{C} 4-\mathrm{C} 5$ & $0.9(7)$ & $\mathrm{C} 11-\mathrm{C} 12-\mathrm{C} 13-\mathrm{C} 14$ & $0.9(8)$ \\
\hline $\mathrm{Br} 1-\mathrm{C} 3-\mathrm{C} 4-\mathrm{C} 5$ & $-177.0(3)$ & $\mathrm{C} 6-\mathrm{C} 1-\mathrm{C} 2-\mathrm{C} 3$ & $-1.4(8)$ \\
\hline $\mathrm{C} 6-\mathrm{C} 5-\mathrm{C} 4-\mathrm{C} 3$ & $0.3(7)$ & $\mathrm{C} 4-\mathrm{C} 3-\mathrm{C} 2-\mathrm{C} 1$ & $-0.3(7)$ \\
\hline $\mathrm{N} 1-\mathrm{N} 2-\mathrm{C} 8-\mathrm{C} 9$ & $-177.5(3)$ & $\mathrm{Br} 1-\mathrm{C} 3-\mathrm{C} 2-\mathrm{C} 1$ & $177.6(4)$ \\
\hline
\end{tabular}

Hydrogen-bond geometry $\left(\AA,{ }^{\circ}\right)$

\begin{tabular}{lllll}
\hline$D-\mathrm{H} \cdots A$ & $D-\mathrm{H}$ & $\mathrm{H} \cdots A$ & $D \cdots A$ & $D-\mathrm{H} \cdots A$ \\
\hline $\mathrm{N} 1-\mathrm{H} 1 N 1 \cdots \mathrm{O} 1^{\mathrm{i}}$ & 0.86 & 2.20 & $3.003(4)$ & 155 \\
$\mathrm{C} 8-\mathrm{H} 8 \cdots \mathrm{O} 1^{\mathrm{i}}$ & 0.93 & 2.42 & $3.234(5)$ & 146 \\
\hline
\end{tabular}

Symmetry code: (i) $x,-y+1 / 2, z+1 / 2$. 